University of Wollongong

Research Online

Faculty of Commerce - Papers (Archive)

Faculty of Business and Law

August 2006

\title{
A Web-based Intelligent Virtual Learning Environment for Industrial Continuous Improvement
}

X. Chi

University of Wollongong

T. A. Spedding

University of Wollongong, spedding@uow.edu.au

Follow this and additional works at: https://ro.uow.edu.au/commpapers

Part of the Business Commons, and the Social and Behavioral Sciences Commons

\section{Recommended Citation}

Chi, X. and Spedding, T. A.: A Web-based Intelligent Virtual Learning Environment for Industrial Continuous Improvement 2006.

https://ro.uow.edu.au/commpapers/274

Research Online is the open access institutional repository for the University of Wollongong. For further information contact the UOW Library: research-pubs@uow.edu.au 


\title{
A Web-based Intelligent Virtual Learning Environment for Industrial Continuous Improvement
}

\author{
Abstract \\ Training in an industrial context is becoming more dynamic and time consuming due to the daily \\ emergence of new technologies and the onset of globalization. People from higher education institutions \\ and industries have applied various heuristic teaching methods in order to inspire students to acquire \\ knowledge in a manufacturing and industrial context. Artificial intelligence techniques has been used for \\ many years in educational systems, however the presentation of domain knowledge is one of the most \\ concerning issues which stems the evolvement of the industrial training systems development. In this \\ paper we present a web-based intelligent virtual learning environment which is designed for the training \\ of industrial continuous improvement techniques. Emphasis is placed on neural network and multi-agent \\ techniques to build state-of-art domain knowledge models which demonstrate realistic industrial \\ environments.

\section{Disciplines} \\ Business | Social and Behavioral Sciences

\section{Publication Details} \\ This paper was originally published as: Chi, X \& Spedding, TA, A Web-based Intelligent Virtual Learning \\ Environment for Industrial Continuous Improvement, IEEE International Conference on Industrial \\ Informatics 2006, Singapore, August 2006, 1102-1107. Copyright 2006 IEEE.
}




\section{A Web-based Intelligent Virtual Learning Environment for Industrial Continuous Improvement}

\author{
Xuesong Chi \\ School of Management and Marketing \\ University of Wollongong \\ Wollongong NSW 2522 \\ AUSTRALIA \\ xc569@uow.edu.au
}

\author{
Trevor A. Spedding \\ School of Management and Marketing \\ University of Wollongong \\ Wollongong NSW 2522 \\ AUSTRALIA \\ spedding@uow.edu.au
}

\begin{abstract}
Training in an industrial context is becoming more dynamic and time consuming due to the daily emergence of new technologies and the onset of globalization. People from higher education institutions and industries have applied various heuristic teaching methods in order to inspire students to acquire knowledge in a manufacturing and industrial context. Artificial intelligence techniques has been used for many years in educational systems, however the presentation of domain knowledge is one of the most concerning issues which stems the evolvement of the industrial training systems development. In this paper we present a web-based intelligent virtual learning environment which is designed for the training of industrial continuous improvement techniques. Emphasis is placed on neural network and multi-agent techniques to build state-of-art domain knowledge models which demonstrate realistic industrial environments.
\end{abstract}

\section{INTRODUCTION}

The ever changing manufacturing environment constantly provides a number of challenges for academia. The most substantial of which, is education. People from higher education institutions and industries have applied various heuristic teaching methods in order to inspire students to gain knowledge in a manufacturing and industrial context. Computer systems play a crucial part when addressing these industrial problems. Techniques such as computer aided instruction (CAI), intelligent tutoring system (ITS), simulation-based training, web-based learning and so on have been developed to assist students to master the knowledge and skills, which are required to further develop and augment their professional careers.

Training in an industrial context is becoming more dynamic and time consuming due to the daily emergence of new technologies and the onset of globalization. Thus computerized training requires more realistic and rapid modelling techniques and training environments. Recently, problem-based learning (PBL) has drawn a lot of attention from educators moving away from traditional theory teaching and case-based learning, as problem-based learning environments use real life problems to provide learners with an authentic learning context.

Computer simulation and virtual environments have been largely used for industrial training in the last twenty years. To build such training environments, the developers need to have a total comprehensive understanding of the problem domain, such as the relationship between the input and output data, in order to interpret and codify the knowledge into the module. The developers must convert this discrete data into procedures the computer can understand. Generally, the environment the student will interact with is predicted and predefined in the system. In these systems the students study the underlying knowledge by following the routines and scenarios which have been preset in the simulation.

Artificial intelligence techniques have been used in learning environments in order to capture the students' response so difficulty and knowledge could dynamically change to adapt to the students' learning pace. Intelligent Tutoring System is a general term to describe educational systems which have a degree of intelligence. It has been used to categorize pedagogical systems which integrate artificial intelligence methodologies to actively assist students to acquire knowledge. On the other hand, many simulation model building environments and learning processes are 'feed-forward'. Intelligence has been applied to many teaching and learning environments. However, intelligence has been mostly used to assist students to perceive the proposed knowledge, or to assist tutors to interpret how students acquire the knowledge. Few researchers have used intelligent learning mechanisms to learn how information from real life cases is represented and interacts with the user. The constructions of existing virtual learning environment models lack many desirable attributes such as flexibility, authenticity, reusability, maintainability or operability. Solving this problem could help developers understand the relationship between simulation input and output.

In this paper we propose $a$ web-based intelligent virtual learning environment (WIVLE), which can be used in higher education institutions and manufacturing environments for training students in industrial quality and process improvement. It is a hybrid intelligent system which incorporates artificial neural networks and agent-based theories. The simulation model is built upon real industrial data used for statistical quality control. This system is built on top of a web-based virtual factory which has been used by the authors to assist the teaching of a university quality engineering course [1].

\section{INTELLIGENT TUTORING SYSTEMS IN INDUSTRY}

Since the first ITS was built in the 1970s, there have been quite a few applications which have been developed to fulfil various pedagogical purposes. SOPHIE [2] is one of the earliest ITS ever built for monitoring students debugging electronic circuits. It evaluates the student's strategy and critiques it according to its own built-in problem solving strategy which is based on the functional specifications of electrical circuits. GUIDON developed by 
Clancy [3] is designed to guide medical students attempting to solve a case of bacteraemia or meningitis, is another famous early tutoring system which uses empirical information to teach students within a problem domain. In the industrial context, very few ITS have been developed [4]. Generally they have been built for teaching mathematics, trouble shooting detection and simple operational training purposes. Reis and Paladini [5] developed an artificial learning environment STCEQ (Sistema Tutorial Inteligente para Controle Estatı'stico da Qualidade - Intelligent Tutoring System for Statistical Quality Control) to teach industrial quality improvement techniques. They use an expert and tutor model to determine the action against the students' answers to the questions. The intelligence in their system was mainly used to diagnose the students response so that their weakness in the topics which could be highlighted and discussed. Another system SAFARI [4] is a project developed by several universities and organizations in Canada in order to facilitate the creation of tutoring systems to be used in professional formation and procedural knowledge teaching. The project provides an approach in building a generic ITS to allow the use of multiple learning strategies. However, a problem emerged when their project became hardware and software platform dependent due to the complexity of the project scope. Therefore network based solutions are preferable for learning, especially in the industrial environment, where hardware and software for training purposes are limited.

It is generally recognized that an ITS consists of four components [6][7]: the domain knowledge module, the pedagogical module, the student module and the interface. However some researchers identify other modules in their ITS, such as an expert module in [8]; an expert, tutorial and problem module in [5] and so on. These modules however can still be categorized under the umbrella of the four main components summarized by Wenger [6] and the major aim of all these modules is to provide sophisticated instructional advice on a one-on-one basis that is comparable to that of a good human tutor [9].

Various artificial intelligent techniques have been used for different ITS research. Typical knowledge-based intelligent paradigms include expert systems, artificial neural networks, fuzzy systems, multiagent based systems, semantic webs and genetic algorithms. Each technique provides a different approach to the learning domains in ITS. Prediction is an important feature of artificial intelligence. Intelligent systems have been used to predict students' behaviour in solving problems [10][11], predicting student's emotions [12] or to predict other system behaviour [13]. Artificial neural networks and associated learning algorithms are popular choices for such tasks, though not the only ones possible. Mohan [14] suggested that linear models are easy to be handled by statistical approaches, whereas it is easier to use neural networks with nonlinear models. However it is hard to find any literature that use nonlinear simulation models in intelligent tutoring systems so that predictions can substitute static simulation algorithm calculation.
The cost and time consumed in developing an ITS is tremendous, leading researchers to continuously search for new methods to save development cost and time. In [8], the authors indicate that 100 hours of development translates into 1 hour of instruction. They suggest that future ITS should be developed with reduced time and cost, and that students should be enabled to work collaboratively. Web-based technologies provide an ideal paradigm for building collaborative working environments, where students can share their learning experiences and discuss their solutions. ELM-ART[11] is one of the web-based tutoring systems which provides interactive learning material and intelligent navigation support, as well as intelligent problem-solving support. The authors suggest that it is relatively easy to port existing intelligent features from ITS to a WWW context.

It is commonly accepted that the aim of ITS is to collaborate with and enhance the learning process. This means that the most effective way so far for students to master the knowledge within a domain is to combine traditional class-based teaching during the "school time" with a realistic tutoring system so that the students can live with the "real" environment and get help without a human teacher. In order to solve the problem between student interpretation of the class taught knowledge and real-life industrial problems, specifically for continuous process improvement, a web-based virtual factory for teaching industrial statistical quality control and continuous improvement techniques has been built by the authors [1]

\section{A WEB-BASED VIRTUAL FACTORY SIMULATOR}

The web-based virtual factory enables students to learn the industrial knowledge collaboratively through a realistic continuously running manufacturing environment. The developed simulator creates a problem-based learning approach with relevant academic underpinning. It provides the student with the experience of applying theory to the practice of operating a factory in real-time. Students are able to "live" with the problem and research over a significant time period and eventually develop solutions. It has always been a problem to teach effectively over the interface between disciplines. This virtual factory addresses this problem and enhances the learning experience by providing a realistic scenario over an extended time frame.

The simulator is interactively controlled over the Internet. Students and lecturers gain access through their group name and password. Each student group have their individual view of the factory under their control. The simulator is initialised with the same conditions by the lecturer. Individual students then access factories to inspect machine status. The lecturer can intervene by creating specific one-off conditions, for example unexpected failures, and obtain an overview of all the groups performances. 


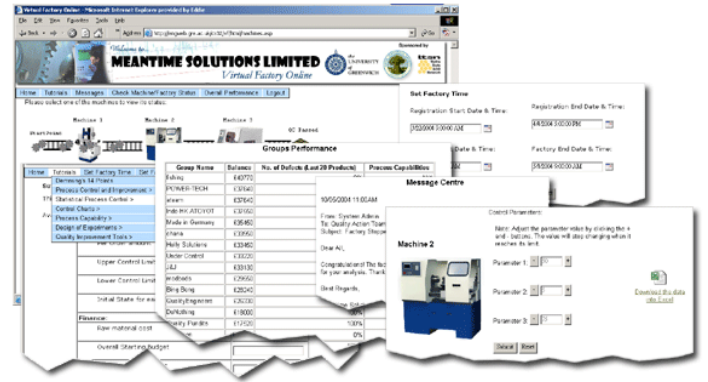

Fig. 1 Interface and Screenshots from the Virtual Factory

This Internet based simulator is platform independent because of its ability to run fully at the server side. As long as the lecturers or students have Internet access and a web browser, they can manage the virtual factory anywhere, anytime. One of the main advantages of using this approach is that the students are able to develop a methodology for quality improvement techniques rather than just an appreciation of the individual tools. Therefore one of the challenges of developing the simulation is to ensure, for example that students are not able to improve a process through experimental design unless they have first got the system in statistical control. The project offers students an opportunity to interact with a realistic scenario over an extended period. This is particularly important to both engineering students who sometimes find it difficult to appreciate the practical relevance of statistics, and statistics students who may find it difficult to appreciate the practical application of their discipline. As demonstrated in Fig. 1, the students and lectures have various interactive interfaces accessible to the virtual factory.

\section{SYSTEM STRUCTURE OF A WEB-BASED INTELLIGENT VIRTUAL LEARNING ENVIRONMENT USING ARTIFICIAL NEURAL NETWORKS AND AGENT-BASED TECHNIQUES}

The web-based virtual factory has been tested and validated by master students studying on a Quality Engineering course at the University of Greenwich in the UK and has received positive responses showing that the students were enthusiastically motivated by the real time operation method. However from a survey among the students, it indicates that the need to develop a more realistic factory environment which includes for example the input and output of real data will benefit especially part-time students from industries to gain hands-on experiences within classes. After evaluation and design, the existing virtual factory has been extended into an intelligent learning environment, which incorporates artificial neural network and multi-agent based technology. The new system is called WIVLE - Web-based Intelligent Virtual Learning Environment. The uniqueness of this system is that the simulation model is built upon real industrial data used for statistical quality control, and integrates novelty detection techniques, so that when the student operates the virtual factory, they will obtain almost the same response output as when they are controlling an real industrial unit. The domain knowledge represented inside the data and the control parameters used to train the neural network is bridged by intelligent agents.

The architecture of the system is shown in Fig. 2, which forms a top down infrastructure. It consists of three tiers: the data tier, modelling tier and application tier. Two intelligent agents collaborate in each tier so that the developer and students can access the modules in each tier for different purposes.

The data tier is on the top of the structure, which provides the real training data required by the neural network to construct the simulation model. It contains two groups of data: Input/Output training data and control data. The input and output data is gathered from the manufacturer and entered by the developer or trainer. Students can also interact with this data to gain an appreciation of how data is used in the simulation model. Control data is set to train the neural network for constructing the simulation model. Only the developers have access to this data.

The modelling tier is composed of a neural network training system. The neural network is based on a back-propagation algorithm. The network is trained and learned by the given data. After teaching the neural network for a certain number of epochs, generalization of the data trend will be summarized and therefore the simulation model is understood by the neural network.

The interface to the learner and the developer, including the existing virtual factory forms the application tier. The virtual factory which was developed earlier as an independent simulation system, acts as a shell and user interaction agent. Two intelligent agents support the virtual factory and liaise between different tiers. The Simulation Construction Agent works as an authoring tool. It leads the trained simulation model to the virtual factory. According to the given virtual factory interface configuration, it fits the model to the learning environment. Then through the interface agent, the students access the virtual factory and 'control' the variables in the factory's machines. The effect on the output will be demonstrated when the input variables are changed by the students. Moreover, the interface agent monitors the students' input, and gives him or her advice according to the learned data from the neural network on the acceptable range of the control parameters,

The system structure complies with the intelligent tutoring system architecture proposed by [6] which is mentioned above. However, this system uses a different approach to model the ITS's domain knowledge module instead of extracting the human tutor's knowledge, it uses a combination of both human knowledge and authentic historical data to construct a "real" industry model so that it can provide students with an appreciation of the real environment in terms of controlling and measuring the realistic parameters as well as a bona fide experience of operating the factory. 


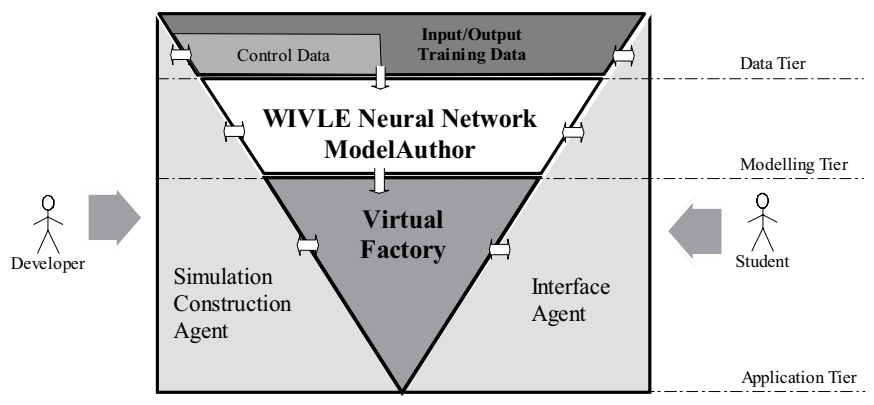

Fig. 2 Architecture for Web-based Intelligent Virtual Learning Environment and interactions between modules and users

\section{A. Artificial Neural Network ModelAuthor (ANNMA)}

Artificial Neural Network (ANN) has been used in solving manufacturing problems for more than two decades. Many artificial intelligent tutoring systems have used ANN in different parts of the system. However, the domain knowledge module, especially in the manufacturing environment has largely depended on the knowledge of the system developer. For instance to build a simulation system for manufacturing process improvement, the relationships between the input parameters and output data need to be understood and represented by the developers. The developers inevitably need to model the distributions of the process data. Special control conditions need to be programmed specifically into the simulation so as to allow the students to identify and solve the problems. So far artificial intelligence has not been applied to help developers develop the domain knowledge of ITS. Based on the pedagogical methodology provided in [1] in teaching statistical quality control techniques, a Back Propagation Neural Network (BPNN) with novelty detection approach is introduced to construct the simulation model.

One may argue that ANN only can be trained correctly if the sample data sets are representative. A key factor that limits the implementation of neural network solutions is the difficulty of validate the reliability of the output generated by the network [15]. In the proposed system, it is necessary to justify sample data sets which feature a very high predictive potential. Therefore in order to train the ANN properly and to present the realistic industrial environment output, the variable data needs to be analysed and classified first. Initially Pearson correlation coefficient is used to test against each input and output to evaluate the relationship between the data sets, as showing in Fig. 3 the data flow diagram of the ANNMA. Since there will be more than one input and output variable, each input set is compared with every output. If the coefficient is larger than the threshold, the input variable is validated to proceed so that it can be used to train the ANN and vice versa.

Bishop [15] demonstrated that novelty data can be quantified by modelling the unconditional probability density of the input data used during training. Once the ANN has been trained by the selected data sets, it will be re-evaluated by the novelty input filter function and the data are fed back to the ANN. By assessing the input variable's unconditional probability density, the degree of novelty of new input vectors can be measured. In this way, the students who give the system unreasonable control can be warned once the density model falls below the threshold assigned to it.

\section{B. Simulation Construction Agent}

One of the important features of WIVLE is the interactive flexible modelling functions provided by the autonomous simulation construction agent (SCA).The SCA provides an interface for the simulation developers to input the data, as well as controlling the data flow from the top of the infrastructure to the bottom to ensure that the domain knowledge is interpreted in a correct way. SCA has the role of the pedagogical component in the WIVLE, where didactical principles can be customized into the environment. There are a few issues in constructing a industrial simulation model in ITS: a) the domain knowledge representation and interpretation; b) the collaboration between knowledge model and the interface; c) the relativity between the input/output data. These issues are addressed by the developers themselves so they can bridge the communication among the modules in ITS. The SCA solves the problem on the developer side by rectifying the flow of information and process, and autonomously proposing the specific modelling adjustment by analysing the abnormalities in the data training set. SCA assists the simulation model building in three phases:

Phase 1: data collection and training

Phase 2: data analysis and special causes classification

Phase 3: variable definitions (e.g. variable name, limits, and intervals)

By passing through these stages with the internal model trained by the ANNMA, SCA collects adequate information for WIVLE to construct the virtual factory simulation. Unlike classical ITS authoring tools, SCA provides better flexibility and less knowledge in using the tool itself. Besides the interaction between the developer and the virtual environment is performed entirely online, the construction of the simulation model will be more transparent to the developers.

\section{Interface agent}

Although the virtual factory provides an interface between the student and the simulation model after the ANNMA and SCA assembles the internal model, the 
interface agent (IA) provides a more comprehensive yet interactive function to the students. It:

1) Demonstrates the training input/output data as examples. Using examples generated and filtered from the real life process training data used in the ANN, students are given a more vivid and visual image on how the process reacts within different scenarios. Then they are allowed to 'touch' the machines themselves after examining the characteristics of the real process.

2) Coordinates the controllers in the interface to represent the real-life scales. At the first stage of the virtual factory, process simulation is generated without the ANNMA. Out-of-control situations occur so that the students can study the underlying knowledge. For example, they can practice the Design of Experiments (DoE) techniques by sliding up and down controllers on the virtual machine. However in reality DoE can only be performed after careful study of the process and often experiments cannot be performed due to the expense and nature of the processes. This will be reflected in the next stage where controls are coordinated by the ANNMA generated model and the IA. The scales and limits are dynamically quantified by the IA through communication to the ANNMA. By examining the trained neural network, it is possible to confine the upper and lower limits so that students gain an appreciation of being aware of the practical range to manipulate the equipments.

3) Records and monitors the student actions for responses and tutoring feedbacks. Using the Novelty Detection techniques in [15], as mentioned in section 4.1, the IA highlights another new notion that effectively evaluates the students' action against the controls. The tutors can manually enlarge the limit on both sides of the control bar, so that the students can try extraordinary input. IA collects the student activities for two uses: one to send the control input data to the Novelty Detection Validator (NDV). If the input is classified as novel, correction advice will be sent to the students; the other usage is to inform the human tutor so that necessary explanations can be carried

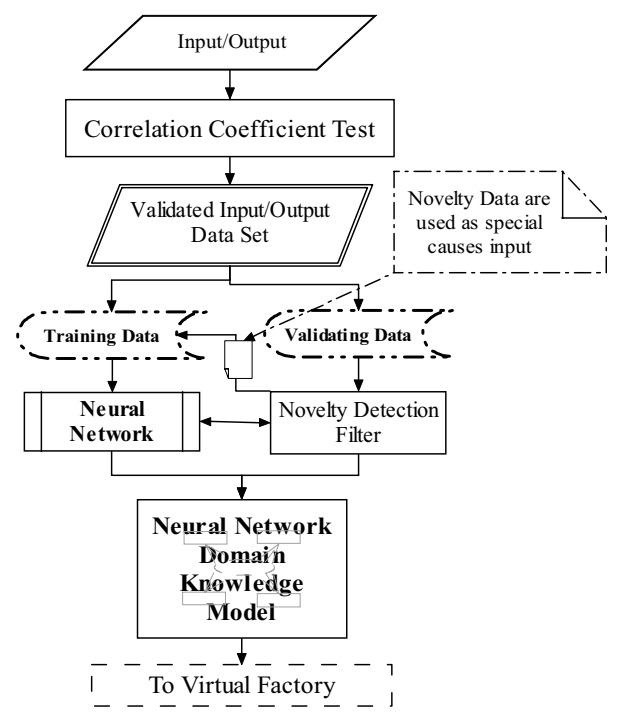

Fig. 3 Artificial Neural Network ModelAuthor out.

\section{CURRENT STATE AND CONSTRAINS OF THE IMPLEMENTATION}

The web-based virtual factory based on simulated data is in its third year of application since its first use in an industrial engineering class. It shows a degree of confidence that this web-based "flight simulator" demonstrates an effective method of allowing students to acquire knowledge through a virtually designed environment.

The implementation of an intelligent extension of the artificial neural network and multi-agent modules are under development. The prototype has been designed for testing purposes. The main constraints to this project concern both industrial practices and the implementation techniques:

1) First, data collection is dependant on the context of the industry. It was found that it is difficult to apply such a training environment in every process industry. High yield manufacturing, plants where data is collected by automated recording equipment or healthcare departments are preferable organizations since their data tends to be standardized and there is a variety of choices of output patterns for training and validation of the neural network.

2) The other constraint is that collected data for training is necessarily presented in a regular pattern due to the nature of neural networks. Although missing data can be detected using the associated learning algorithm to fit into the model, novelty input from outside the training range is still treated as extraordinary which means the associated output is unpredictable and has to be pinpointed or discarded.

The need for a realistic environment training tool is increasing. An intelligent interactive learning environment which reproduces the actual scenario of an industry could be a solution to the difficulties of contemporary engineering education, and we consider this approach to be very important.

\section{CONCLUSION}

A web-based virtual learning environment to teach industrial continuous improvement techniques has been discussed in this paper. This system integrates various artificial intelligent techniques including neural networks and intelligent agents. This highlights the use of real industrial data in building a simulation environment so that students can appreciate a realistic training scenario without accessing the actual plant or loosing the interaction of learning underlying engineering knowledge. The website serves as a platform independent feature which also facilitates collaboration in the learning process, where students learn through discussion and competition.

Upon acquiring the proper knowledge using the simulated data, the students will move onto a synthetic process, which can be treated as a real time system with connections to the machinery. Further this environment can be used as an effective predictor of the process optimization, and will eventually have the ability to connect to real online systems. 


\section{ACKNOWLEDGMENT}

The authors would like to express their appreciations to the Royal Statistical Society Centre for Statistical Education, the Maths, Stats and OR network, UK, the Medway School of Engineering, University of Greenwich, UK and School of Management and Marketing, University of Wollongong, Australia for jointly supporting this work.

\section{REFERENCES}

[1] X. Chi, M. P. J. Pepper, and T. A. Spedding, "A Web-based Virtual Factory and Simulator for Industrial Statistics," in Proceeding of the 2004 Winter Simulation Conference, Washington D.C., USA, 2004, pp. 2103-2106.

[2] J. S. Brown, R. R. Burton, and A. G. Bell, "SOPHIE: a sophisticated instructional environment for teaching electronic troubleshooting," in BBN Report 2790. Cambridge, Massachusetts: Bolt Beranek and Newman Inc., 1974.

[3] W. J. Clancey, "Tutoring rules for guiding a case method dialogue," in Intelligent tutoring systems, D. S. J. S. Brown, Ed. New York: Academic Press, 1982, pp. 201-226.

[4] C. Frasson and E. Aimeur, "Designing a multi-strategic intelligent tutoring system for training in industry," Computers in Industry, vol. 37, no. 2, 1998, pp. 153-167.

[5] M. M. Reis, E. P. Paladini, S. Khator, and W. A. Sommer, "Artificial intelligence approach to support statistical quality control teaching," Computers \& Education, In Press, Corrected Proof, no., 2005.

[6] E. Wenger, Artificial intelligence and tutoring systems: computational and cognitive approaches to the communication of knowledge. Morgan Kaufmann Publishers, Los Altos, Calif: 1987, p.486.

[7] B. Woolf, "AI in Education," in Encyclopedia of Artificial Intelligence, S. Shapiro, Ed. New York: John Wiley \& Sons, Inc., 1992, pp. 434-444.

[8] J. Beck, M. Stern, and E. Haugsjaa, "Applications of AI in education," Crossroads, vol. 3, no. 1, 1996, pp. 11-15.

[9] P. Sedlmeier, J. S. Neil, and P. B. Baltes, "Intelligent Tutoring Systems," in International Encyclopedia of the Social \&Behavioral Sciences. Oxford: Pergamon, 2001, pp. 7674-7678

[10] T. Murray, "Authoring Intelligent Tutoring Systems: An analysis of the state of the art," International Journal of Artificial Intelligence in Education, vol. 10, no., 1999, pp. 98-129.

[11] P. Brusilovsky, E. Schwarz, and G. Weber, "ELM-ART: An Intelligent Tutoring System on World Wide Web," in Proceeding of the Intelligent Tutoring Systems Conference, 1996, pp. 261-269.

[12] D. J. Litman and S. Silliman, "ITSPOKE: An Intelligent Tutoring Spoken Dialogue System," in Proceeding of the Human Language Technology Conf.: 4th Meeting of the North American Chap. of the Assoc. for Computational Linguistics (HLT/NAACL), 2004, pp. 233-236.
[13] D. Benyon and D. Murray, "Adaptive systems: from intelligent tutoring to autonomous agents," Knowledge Based Systems, vol. 6, no. 4, 1993, pp. 197.

[14] C. K. Mohan, Frontiers of expert systems: reasoning with limited knowledge. Kluwer Academic Publishers: 2000.

[15] C. M. Bishop, "Novelty detection and neural network validation," IEE Proc.-Vis. Image Signal Process., vol. 141, no. 4, 1994, pp. 217-222. 